\title{
On flow of a heat conducting fluid through a porous medium
}

\author{
S. J. Jacobs Department of Atmospheric and Oceanic Science, and Department of \\ Mechanical Engineering and Applied Mechanics, The University of Michigan, Ann Arbor, \\ MI 48107, USA
}

Accepted 1986 March 26. Received 1986 March 26; in original form 1985 December 26

\begin{abstract}
Summary. A two-space singular perturbation technique is employed to derive approximate governing equations for flow of a viscous heat-conducting fluid through a rigid porous solid. It is assumed that buoyancy forces are significant, and it is shown that standard approximations used in the study of flow through a porous medium are valid provided that $\mathrm{Gr} \ll 1$, where $\mathrm{Gr}$ is a Grash of number calculated using a typical pore radius as the length scale. Results previously derived in the literature for flow through an isotropic random array of spherical particles are used to show how the permeability and conductivity tensors can be calculated for a problem of interest in planetary science.
\end{abstract}

Key words: bulk material properties, porous media, effective properties of heterogeneous media

\section{Introduction}

Determining effective heat and mass transport properties for fluid flow and heat transfer in a porous medium is an important problem in the study of geothermal systems (Cheng 1978), soil consolidation (Biot 1955), climate changes in the Martian atmosphere (Fanale et al. 1982), and a number of other cases of interest in geophysics and planetary science. The most widely used approach for deriving approximate theories for the treatment of flow through a porous medium involves the use of a local averaging technique (Gray 1975; Gray \& O'Neill 1976; Whitaker 1977; Hassanizadeh \& Gray 1979). Unfortunately, the equations obtained through use of the method are incomplete in the sense that direct measurements are needed to provide the values of various constants which enter into the analysis. Continuum mechanics theories of the type developed by Truesdell and others (Truesdell 1984) suffer from the same drawback, and the probability approach reviewed by Batchelor (1974) is difficult to apply in most cases.

Measurement of effective transport properties is seldom easy, and in some cases is prohibitively difficuit. Consequently, much effort has been devoted to relating bulk 
properties to the small-scale structure and character of the medium. A number of such studies have been carried out for the case of constant pressure and temperature gradients, and will be cited later in the text. For practical purposes it is necessary to relate such studies to the more general case in which the gradients of macroscopic properties are non-uniform. Since the macroscopic problem is usually characterized by a length scale large compared to the length scale of the inhomogeneities, a singular perturbation treatment based on the use of a two-space approach appears to be the most logical method for deriving the macroscopic equations and for determining effective bulk material properties. The two-space procedure is compatible with the averaging method, and can be understood as a technique for evaluating integrals appearing in the latter method by expressing them in terms of the solution of a small-scale problem.

Investigations employing this method have been carried out in the study of slow flow of a viscous barotropic fluid through a porous medium (Keller 1980), of the equations of poroelasticity (Burridge \& Keller 1981; Sanchez-Palencia 1980), and, in an informal application of the method, of Fickian diffusion in a porous medium (Carbonell \& Whitaker 1983). The purpose of the present study is to generalize this work by considering coupled heat and mass transfer for flows such that the buoyancy force in the hydrodynamic momentum equation is significant in driving the motion. A scale analysis of the hydrodynamic equations for this case indicates that the Grashof number calculated using a typical pore radius as a length scale serves as the Reynolds number for the small-scale flow. Examples are cited to show that the Grashof number is small in all cases of practical interest, and this result is used in deriving the equations which govern the large-scale flow.

In addition to deriving these equations, we establish general properties of the permeability and conductivity tensors. We also use the results mentioned in the previous paragraph for the uniform gradient case to indicate how these tensors can be calculated for flow through an isotropic random array of spherical particles, which appears to be a reasonable model for determining transport properties of the Martian regolith in studies of the type carried out by Fanale et al. Application of the results derived here is reserved for future study.

\section{Formulation}

We consider flow of a viscous fluid through a rigid porous solid, and we use the Boussinesq approximation in treating the hydrodynamic equations. Let $\mathrm{x}=(x, y, z)$ denote the position vector, $\theta$ the difference between the temperature in the solid and a reference temperature, $T$ the temperature difference in the fluid, $p$ the fluid pressure, $\mathbf{u}$ the fluid velocity, $\rho$ the fluid density evaluated at the reference temperature, $g$ the gravitational vector, $V_{\mathrm{f}}$ the volume occupied by the fluid, $V_{\mathrm{s}}$ the volume occupied by the solid, and $A$ the boundary between the fluid and the solid. Then, taking the $z$-axis as the vertical direction and $\hat{\mathbf{n}}$ as the unit normal to $A$ pointing into the solid, the governing equations are

$$
\begin{aligned}
& \nabla \cdot \mathbf{u}=0 \\
& \frac{D \mathbf{u}}{D t}+\frac{1}{\rho} \nabla p=\nu \nabla^{2} \mathbf{u}-g \mathbf{k}(1-\beta T), \\
& c_{\mathrm{f}} \frac{D T}{D t}=k_{\mathrm{f}} \nabla^{2} T,
\end{aligned}
$$


in $V_{\mathrm{f}}$,

$c_{\mathrm{s}} \frac{\partial \theta}{\partial t}=k_{\mathrm{s}} \nabla^{2} \theta$

in $V_{\mathrm{s}}$, and

$\mathbf{u}=0, \quad k_{\mathbf{f}} \hat{\mathbf{n}} \cdot \nabla T=k_{\mathrm{s}} \hat{\mathbf{n}} \cdot \nabla \theta, T=\theta$,

on $A$. It is assumed that the kinematic viscosity $\nu$, the coefficient of thermal expansion $\beta$, the specific heats at constant pressure per unit volume $c_{\mathrm{f}}$ and $c_{\mathrm{s}}$, and the thermal conductivities $k_{\mathrm{f}}$ and $k_{\mathrm{s}}$ are constants, and that thermal expansion of the solid can be neglected.

We now define a characteristic length scale $h$ for the pores, a macroscopic length scale $H$, a velocity scale $U$, and a temperature scale $\Delta T$, with $h \leqslant H$. Introducing the scaled variables

$\mathbf{x}_{*}=\mathrm{x} / H, \quad t_{*}=\nu t / H^{2}, \quad p_{*}=\left[h^{2} /(\rho \nu U H)\right](p+\rho g z)$,

$\mathbf{u}_{*}=\mathbf{u} / U, \quad T_{*}=T / \Delta T, \quad \theta=\theta / \Delta T$,

and expressing the governing equations in terms of the scaled variables yields a dimensionless form of these equations in which

$\epsilon=h / H$

appears as a small parameter. The characteristic pore radius is $O(\epsilon)$ when expressed in dimensionless form, and so, omitting asterisks, the dependent variables are functions of both $\mathbf{x}$ and

$\mathbf{y}=\mathbf{x} / \epsilon$.

To treat the equations for this case we use a two-space method in which $\mathbf{x}$ and $\mathbf{y}$ are regarded as independent variables. In the present study we assume that the pores form a periodic array, that all functions $f(\mathbf{x}, \mathbf{y}, t)$ are periodic in $\mathbf{y}$, and that all surfaces $A$ separating the fluid and solid in a periodic cell are closed. The slowly varying part of $f$ can then be determined by averaging $f$ over a periodic cell.

In the two-space approach the gradient operator is given by

$\nabla=\nabla_{x}+\epsilon^{-1} \nabla_{y}$,

and the dimensionless equations of motion become

$\nabla_{y} \cdot \mathbf{u}+\epsilon \nabla_{x} \cdot \mathbf{u}=0$,

$\epsilon^{2} \frac{\partial \mathbf{u}}{\partial t}+\epsilon R \mathbf{u} \cdot\left(\nabla_{y} \mathbf{u}+\epsilon \nabla_{x} \mathbf{u}\right)+\frac{1}{\epsilon} \nabla_{y} p+\nabla_{x} p=D^{2} \mathbf{u}+B \hat{\mathbf{k}} T$,

$\epsilon^{2} \frac{\partial T}{\partial t}+\epsilon R \mathbf{u} \cdot\left(\nabla_{y} T+\epsilon \nabla_{x} T\right)=\frac{1}{\sigma_{\mathrm{f}}} D^{2} T$,

$\epsilon^{2} \frac{\partial \theta}{\partial t}=\frac{1}{\sigma_{\mathrm{s}}} D^{2} \theta$

together with the boundary conditions

$\mathbf{u}=0, \quad \hat{\mathbf{n}} \cdot\left(\nabla_{y} T+\epsilon \nabla_{x} T\right)=\alpha \hat{\mathbf{n}} \cdot\left(\nabla_{y} \theta+\epsilon \nabla_{x} \theta\right), \quad T=\theta$,

in which $D^{2}$ is the Laplacian operator given by

$D^{2}=\nabla_{y}^{2}+2 \epsilon \nabla_{y} \cdot \nabla_{x}+\epsilon^{2} \nabla_{x}^{2}$. 
In these equations the ratio of thermal conductivities $\alpha$ and the Prandtl numbers $\sigma_{\mathrm{f}}$ and $\sigma_{\mathrm{s}}$ are defined by

$\alpha=k_{\mathrm{s}} / k_{\mathrm{f}}, \quad \sigma_{\mathrm{f}}=\left(\nu c_{\mathrm{f}}\right) / k_{\mathrm{f}}, \quad \sigma_{\mathrm{s}}=\left(\nu c_{\mathrm{s}}\right) / k_{\mathrm{s}}$,

and the Reynolds number $\mathrm{R}$ and buoyancy parameter $B$ are defined as

$\mathrm{R}=r / \epsilon, \quad B=U_{\mathrm{T}} / U$,

in which

$r=U h / \nu, \quad U_{\mathrm{T}}=\left(\beta g \Delta T h^{2}\right) / \nu$,

denote a Reynolds number based on the pore length scale and a free convection velocity scale.

Flow in a porous medium is driven by a combination of buoyancy and mechanical forces, for which the velocity scales are given by $(2.18)$ and by $U_{\mathrm{M}}$, a mechanical velocity scale associated with an imposed pressure gradient or the motion of distant boundaries. In the forced convection case, described mathematically by $U_{\mathrm{T}} \ll U_{\mathrm{M}}$, the appropriate choice for the velocity scale is $U=U_{\mathrm{M}}$, and the buoyancy force can be neglected in the treatment of the small-scale dynamics. Here we consider mixed free and forced convection, and accordingly we take $U=U_{\mathrm{T}}$. Then $B=1$, and the small-scale Reynolds number is given by $\mathrm{r}=\mathrm{Gr}$, where

$\mathrm{Gr}=\left(\beta g \Delta T h^{3}\right) / \nu^{2}$,

is a Grashof number based on the length scale of the pores. Previous studies of flow in a porous medium show that Darcy's law is applicable only for small values of the small-scale Reynolds number, and therefore we must determine the magnitude of the Grashof number for flows of practical interest.

To provide examples of the magnitude of the Grashof number, we consider two cases of interest, the flow of carbon dioxide through the assemblage of small boulders which make up the Martian regolith, and the flow water through high-permeability sandstone in a terrestrial geological formation. Using currently accepted values for the physical properties of interest, we find that

$\beta=0.004 \mathrm{~K}^{-1}, \quad g=376 \mathrm{~cm}^{2} \mathrm{~s}^{-1}$,

$\nu=7 \mathrm{~cm}^{2} \mathrm{~s}^{-1}, \quad h=0.1 \mathrm{~cm}$,

for the Martian case, and

$\beta=2 \times 10^{-4} \mathrm{~K}^{-1}, \quad g=981 \mathrm{~cm}^{2} \mathrm{~s}^{-1}$,

$\nu=0.01 \mathrm{~cm}^{2} \mathrm{~s}^{-1}, \quad h=0.001 \mathrm{~cm}$,

for flow through terrestrial sandstone, which yields

$\mathrm{Gr}_{\mathrm{Mars}}=(\Delta T) /\left(3 \times 10^{4} \mathrm{~K}\right), \quad \mathrm{Gr}_{\text {Earth }}=(\Delta T) /\left(5 \times 10^{5} \mathrm{~K}\right)$.

As indicated by (2.21), $\mathrm{Gr} \ll 1$ in all cases of physical interest, and since $\epsilon$ is also small, the governing equations can be solved by expanding the dependent variables in powers of both small parameters. Carrying out such an expansion and working to lowest order in $\mathrm{Gr}$ yields the same solutions as those obtained by assuming that $\mathrm{Gr}=0(\epsilon)$. Therefore, $(2.10)-$ (2.14) will be treated for the case $\epsilon \ll 1$ with $B=1$ and with $\mathrm{R}$ assumed to be an $O(1)$ quantity. 
We conclude this section by noting that in applications of the two-space method the dependence of the dependent variables on the slow space scale $x$ is chosen to eliminate secularities in the dependence of these variables on the fast scale $y$. In continuum mechanics studies the relations which eliminate secularities can be obtained most efficiently by averaging the equations of motion over a domain in $y$-space. This method will be applied in the next section to derive the equations governing the large-scale motion.

\section{Analysis}

Expanding $T$ in the form

$T=T_{0}+\epsilon T_{1}+\ldots$,

and substituting into (2.12) shows that $T_{0}$ is independent of $\mathbf{y}$. Thus, turning to the continuity and momentum equations (2.10) and (2.11) and expanding the other dependent variables in the form $(3.1)$, we obtain

$p_{0}=p_{0}(\mathbf{x}, t)$,

$\nabla_{y} \cdot \mathbf{u}_{0}=0$,

$\nabla_{y} p_{1}=\nabla_{y}^{2} \mathbf{u}_{0}-\left(\nabla_{x} p_{0}-T_{0} \hat{\mathbf{k}}\right)$

and

$\nabla_{y} \cdot \mathbf{u}_{1}+\nabla_{x} \cdot \mathbf{u}_{0}=0$.

Because (3.3) and (3.4) are linear, the solution for $\nabla_{y} p_{1}$ and $\mathbf{u}_{0}$ can be expressed in the form

$\mathbf{u}_{0}=-\mathrm{U}(\mathbf{y}) \cdot\left(\nabla_{x} p_{0}-T_{0} \hat{\mathbf{k}}\right), \quad \nabla_{y} p_{1}=-\left(\nabla_{y} \pi\right) \cdot\left(\nabla_{x} p_{0}-T_{0} \hat{\mathbf{k}}\right)$,

in which $\pi$ is a vector, $U$ is a second-order tensor, and tensor notation (Bird et al. 1977, appendix $\mathrm{A}$ ) is used in computing the $y$-gradient of $\pi$. Here $\pi$ and $U$ solve

$\nabla_{y} \cdot \mathrm{U}=0$,

$\nabla_{y} \pi=\nabla_{y}^{2} \mathrm{U}+\mathrm{I}$,

where $\mathrm{I}$ is the unit tensor and where $\mathrm{U}$ vanishes on $A$ and is periodic in $\mathrm{y}$.

In engineering applications the average velocity is usually defined as a superficial average rather than an interstitial mean. Therefore, we define superficial averages $w$ and $W$ of $u_{0}$ and $U$ through the relations

$\mathbf{w}=\frac{1}{V_{\mathrm{t}}} \int \mathbf{u}_{0} d V, \quad \mathrm{w}=\frac{1}{V_{\mathrm{t}}} \int \mathrm{U} d V$,

where the integrals are taken over the fluid part of a periodic cell and where $V_{\mathrm{t}}$ is the total volume of the cell. The properties of $W$ can be determined by dotting $U^{T}$ into (3.8), averaging, and expressing the result in component form. Use of the divergence theorem and the boundary and periodicity conditions yields

$W_{m k}=\left[\left(\nabla_{y} U_{i m}\right) \cdot\left(\nabla_{y} U_{i k}\right)\right]$,

where the brackets denote the average. It can be seen by inspection that $W$ is symmetric, and therefore a set of axes exists in which $W$ is diagonal. Expressing (3.10) in this set of axes shows that the diagonal elements of $W$ are positive, and so $W$ is positive definite. 
This result was stated without proof in a paper by Saffman (1971) concerning boundary conditions for flow in a porous medium.

Averaging (3.5) and (3.6) and noting that the average of the first term in (3.5) vanishes because of the boundary and periodicity conditions, we obtain

$\mathbf{w}=-\mathbf{w} \cdot\left(\nabla_{x} p_{0}-T_{0} \hat{\mathbf{k}}\right)$,

and

$\nabla_{x} \cdot \mathrm{w}=0$.

Equations (3.11) and (3.12) provide a generalized form of Darcy's law, in which the permeability tensor $W$ is a positive definite symmetric matrix. If the material is isotropic on the small scale, $W$ is a positive scalar multiple of the unit tensor.

It is convenient before treating the temperature equations to multiply $(2.12)$ by $\sigma_{f}$ and to integrate the result over the fluid part of the volume of a periodic cell, to multiply (2.13) by $\alpha \sigma_{s}$ and to integrate over the solid part of the cell, and to add the two in tegrals. Dividing the result by $\epsilon^{2}$ and using the second of the boundary conditions $(2.14)$ yields

$$
\begin{aligned}
\frac{\partial}{\partial t}\left(\sigma_{\mathrm{f}} \int T d V+\alpha \sigma_{\mathrm{s}} \int \theta d V\right)+R \sigma_{\mathrm{f}} \nabla_{x} \cdot \int(\mathbf{u} T d V)= & \nabla_{x}^{2}\left(\int T d V+\alpha \int \theta d V\right) \\
& +\epsilon^{-1} \int \hat{\mathbf{n}} \cdot\left(\nabla_{x} T-\alpha \nabla_{x} \theta\right) d A .
\end{aligned}
$$

To treat (2.12) and (2.13), we expand $T$ and $\theta$ in powers of $\epsilon$, and obtain

$T_{0}=T_{0}(\mathbf{x}, t), \quad \theta_{0}=T_{0}(\mathbf{x}, t)$,

and

$\nabla_{y}^{2} T_{1}=0, \quad \nabla_{y}^{2} \theta_{1}=0$

along with the boundary conditions

$\hat{\mathbf{n}} \cdot\left(\nabla_{y} T_{1}-\alpha \nabla_{y} \theta_{1}\right)=(\alpha-1) \hat{\mathbf{n}} \cdot \nabla_{x} T_{0}, \quad T_{1}=\theta_{1}$

on $A$. The equations for $T_{1}$ and $\theta_{1}$ are linear, and so these variables can be expressed in the form

$T_{1}=\mathbf{G}(\mathbf{y}) \cdot \nabla_{x} T_{0}+\psi(\mathbf{x}, t), \quad \theta_{1}=H(\mathbf{y}) \cdot \nabla_{x} T_{0}+\psi(\mathbf{x}, t)$,

where $\mathbf{G}$ and $\mathbf{H}$ are vectors satisfying Laplace's equation in their domains of definition, $\psi$ is an arbitrary additive function, and $\mathbf{G}$ and $\mathbf{H}$ satisfy

$\hat{\mathbf{n}} \cdot \nabla_{y}(\mathbf{G}-\alpha \mathbf{H})=(\alpha-1) \hat{\mathbf{n}}, \quad \mathbf{G}=\mathbf{H}$,

on $A$ and a periodicity condition.

Properties of the second-order tensor

$\mathrm{S}=\frac{1}{V_{\mathrm{t}}} \int \hat{\mathbf{n}} \mathbf{G} d A$

can be obtained as follows. Multiply the Laplace equations satisfied by $\mathbf{G}$ and $\mathbf{H}$ by $\mathbf{G}$ and $\alpha \mathbf{H}$, respectively, integrate the resulting equations over the volumes occupied by the fluid and solid in a periodic cell, and apply the boundary and periodicity conditions. Use of the 
divergence theorem then shows that

$(\alpha-1) S_{m k}=\left\{\int \nabla\left(\nabla_{y} G_{m}\right) \cdot\left(\nabla_{y} G_{k}\right) d V+\alpha\left[\left(\nabla_{y} H_{m}\right) \cdot\left(\nabla_{y} H_{k}\right) d V\right\} / V_{\mathrm{t}}\right.$,

which in turn implies that $(\alpha-1) \mathrm{S}$ is symmetric and positive definite if $\alpha \neq 1$.

We now substitute (3.14) and (3.17) into (3.13), and we note that the contribution to this expression involving the additive function $\psi$ in (3.17) is proportional to the integral of $\hat{\mathrm{n}}$ over the closed area $A$, and therefore vanishes. Dividing (3.13) by $V_{\mathrm{t}}$ and letting

$\phi_{\mathrm{f}}=V_{\mathrm{f}} / V_{\mathrm{t}}, \quad \phi_{\mathrm{s}}=V_{\mathrm{s}} / V_{\mathrm{t}}$,

denote the volume fractions of the fluid and solid, we find that (3.13) becomes

$\left(\sigma_{\mathrm{f}} \phi_{\mathrm{f}}+\alpha \sigma_{\mathrm{S}} \phi_{\mathrm{s}}\right) \frac{\partial T_{0}}{\partial t}+\sigma_{\mathrm{f}} R \mathbf{w} \cdot \nabla_{x} T_{0}=\nabla_{x} \cdot\left\{\left(\phi_{\mathrm{f}}+\alpha \phi_{\mathrm{s}}\right) \mid-(\alpha-1) \mathrm{S}\right\} \cdot \nabla_{x} T_{0}$.

The conductivity tensor, given in (3.22) by the expression in curly brackets, is the difference between two symmetric positive definite tensors. This is consistent with a result derived using a probability model (Batchelor 1974) concerning the magnitude of the bulk conductivity for the isotropic case. On physical grounds we expect that the conductivity tensor is positive definite, but this has been proved only for the isotropic case, for which the conductivity tensor is a multiple of the unit tensor and for which a variational principle (Hashin \& Strikman 1962) provides positive upper and lower bounds for this multiple.

We now express the foregoing results in dimensional form. Let $u$ and $p$ denote the lowest order contributions to the average velocity and the pressure, $T$ the lowest order contribution to the difference between the temperature and a reference temperature, and $\nabla$ and $\partial / \partial t$ the dimensional gradient and time derivative, and define the dimensional permeability tensor $\mathrm{N}$ by

$\mathrm{N}=\frac{h^{2}}{\rho \nu} \mathrm{W}$

the effective specific heat $c$ by

$c=c_{\mathrm{f}} \phi_{\mathrm{f}}+c_{\mathrm{s}} \phi_{\mathrm{s}}$,

and the dimensional conductivity tensor $K$ by

$\mathrm{K}=\left(k_{\mathrm{f}} \phi_{\mathrm{f}}+k_{\mathrm{s}} \phi_{\mathrm{s}}\right) \mathrm{I}-\left(k_{\mathrm{s}}-k_{\mathrm{f}}\right) \mathrm{S}$,

where $W$ and $S$ are the dimensionless tensors defined previously. Then the approximations to the equations governing mass and heat transfer in a porous medium become

$\mathbf{u}=-\mathrm{N} \cdot\{\nabla p+\rho g(1-\beta T) \hat{\mathbf{k}}\}$,

$\nabla \cdot \mathbf{u}=0$,

and

$c \frac{\partial T}{\partial t}+c_{\mathrm{f}} \mathbf{u} \cdot \nabla T=\nabla \cdot(\mathrm{K} \nabla T)$

In the case of diffusion of a solute through fluid in the pores of an impermeable solid, the mass fraction $q$ of the solute satisfies (3.28) with $T$ replaced by $q, c$ by unity, $k_{\mathrm{f}}$ by the Fickian diffusion constant $D$, and $k_{\mathrm{s}}$ by zero.

As shown by Burridge \& Keller (1981), the two-space approach can also be applied when 
the medium is not periodic on the small scale. However, detailed calculations of the permeability and conductivity tensors are available only for mass and heat transfer through a periodic isotropic array of spheres of equal radius. These calculations were carried out assuming constant macroscopic pressure and temperature gradients, but use of the two-space method casts the small-scale problem in a form for which results derived assuming constant macroscopic gradients can be used to determine the permeability and conductivity tensors.

Numerical calculations (Sangani \& Acrivos 1982; Zick \& Homsy 1982) show that the permeability tensor $\mathbf{N}$ for flow through an isotropic spherical array reduces to a multiple $n$ of the unit tensor, where

$n=2 a^{2} /\left(9 \rho \nu \phi_{\mathrm{s}} \Gamma\right)$,

in which $a$ is the radius of the spheres and $\Gamma$ is a constant which depends on the properties of the array. Sangani \& Acrivos suggest use of the values $\Gamma=87, \phi_{\mathrm{s}}=0.62$, for treating random closely packed arrays. The bulk thermal conductivity for heat transfer in an array of this type is a multiple $k$ of the unit tensor, and has been calculated in a number of papers, most recently by Sangani \& Acrivos (1983). Sangani \& Acrivos provide values of the conductivity as a function of $\alpha$ for closely packed random arrays in their table 2 , and also confirm the accuracy of an asymptotic expression for the bulk conductivity valid for large values of $\alpha$ derived by Batchelor \& O'Brien (1977). The numerical values for the effective conductivity can also be used to treat the case of Fickian diffusion by making the substitutions indicated in the sentence following (3.28).

As indicated previously, we regard a random array of closely packed spheres as providing a reasonable model for study of transport in the Martian regolith, and consequently the theory derived above provides results which can be applied in the study of a problem of practical interest in planetary studies.

\section{Concluding remarks}

The purpose of theories of the present type is to derive a set of approximate equations describing mass and heat transfer in a heterogeneous medium in a manner which displays the parameter ranges for which the approximation is valid and which shows how bulk properties of the medium can be calculated by solving a suitably posed small-scale problem. The present calculation is fairly simple because the small-scale motion can be treated using a linear theory. Other examples of cases for which the two-space approach can be applied are the theory of thermoelasticity in a porous medium, which was studied using a phenomenological theory by Morland (1978), and hydrothermal convection in mid-ocean ridges (Fehn \& Cathles 1979). Non-linear problems such as the interaction between large- and smallscale oceanic eddies (Pedlosky 1984) or the effect of clouds on the general circulation of the atmosphere are more in teresting but are far more difficult to treat.

\section{Acknowledgment}

The author wishes to thank Professor William Kuhn for calling his attention to this problem.

\section{References}

Batchelor, G. K., 1974. Transport properties of two-phase materials with random structure, Ann. Rev. Fluid Mech., 6, 227-255.

Batchelor, G. K. \& O'Brien, R. W., 1977. Thermal or electrical conduction through a granular material, Proc. R. Soc. A, 355, 313-333. 
Biot, M. A., 1955. Theory of elasticity and consolidation for a porous anisotropic solid, J. appl. Phys., 26, 182-185.

Bird, R. B., Hassager, O., Armstrong, R. C. \& Curtiss, C. F., 1977. Dynamics of Polymeric Liquids, I, Wiley, New York.

Burridge, R. \& Keller, J. B., 1981. Poroelasticity equations derived from microstructure, J. acoust. Soc. Am., 70, 1140-1146.

Cheng, P., 1978. Heat transfer in geothermal systems, Adv. Heat Transfer, 14, 1-105.

Carbonell, R. G. \& Whitaker, S., 1983. Dispersion in pulsed systems - II, Chem. Engng. Sci., 38, 17951802 .

Fanale, F. P., Salvail, J. R., Banerdt, W. B. \& Saunders, R. S., 1982. Mars: The regolith-atmosphere-cap system and climate change, Icarus, 50, 381-407.

Fehn, U. \& Cathles, L. M., 1979. Hydrothermal convection at slow-spreading mid-ocean ridges, Tectonophys., 55, 239-260.

Gray, W. G., 1975. A derivation of the equations for multi-phase transport, Chem. Engng. Sci, 30, 229-233.

Gray, W. G. \& O'Neill, K., 1976. On the general equations for flow in porous media and their reduction to Darcy's law, Wat. Resour. Res., 12, 148-156.

Hashin, Z.\& Strikman, S., 1962. A variational approach to the theory of the effective magnetic permeability of multiphase materials, J. appl. Phys., 33, 3125-3131.

Hassanizadeh, M. \& Gray, W. G., 1979. General conservation equations for multi-phase systems: 1. Averaging procedure, Adv. Wat. Resour., 2, 131-144.

Keller, J. B., 1980. Darcy's law for flow in porous media and the two-space method, in Nonlinear Partial Differential Equations in Engineering and Applied Science, pp. 429-443, eds Sternberg, R. L. Kalinowski, A. J. \& Papadakis, J. S., Marcel Dekker, New York.

Morland, L. W., 1978. A theory of slow fluid flow through a porous thermoelastic matrix, Geophys. J. R. astr. Soc., 55, 393-410.

Pedlosky, J., 1984. The equations for geostrophic motion in the ocean, J. phys. Oceangr., 14, 448-455.

Saffman, P. G., 1971. On the boundary conditions at the surface of a porous medium, Stud. appl. Math., 50, 93-101.

Sanchez-Palencia, E., 1980. Non-homogeneous media and vibration theory, in Lecture Notes in Physics, 127, Springer-Verlag, New York.

Sangani, A. S. \& Acrivos, A., 1982. Slow flow through a periodic array of spheres, Int. J. multiphase Flow, 8, 343-360.

Sangani, A. S. \& Acrivos, A., 1983. The effective conductivity of a periodic array of spheres, Proc. $R$. Soc. $A$, 386, 263-275.

Truesdell, C., 1984. Rational Thermodynamics, 2nd ed, Springer-Verlag, New York.

Whitaker, S., 1977. Simultaneous heat, mass, and momentum transfer in porous media, Adv. Heat Transfer, 13, 119-203.

Zick, A. A. \& Homsy, G. M., 1982. Stokes flow through periodic arrays of spheres, J. Fluid Mech., 115, 13-26. 